Adenine phosphoribosyltransferase (APRT)

4-amino-5-imidazolecarboxamide (AICA)

5-amino-1-ribosyl-4-imidazolecarboxamide (rAICA) erythrocyte hypoxanthine-guanine phosphoribosyltransferase (HGPRT) inosine 5'-phosphate (IMP)

Lesch-Nyhan syndrome

5'-phosphoribosyl-5-aminoimidazole-4-carboxamide (AICAR)

\title{
Lesch-Nyhan Syndrome: The Synthesis of Inosine 5'-Phosphate in the Hypoxanthine-Guanine Phosphoribosyltransferase-deficient Erythrocyte by Alternate Biochemical Pathways
}

\author{
BERTRAM A. LOWY(3") AND MARJORIE K. WILLIAMS \\ Department of Biochemistry, Albert Einstein College of Medicine, Bronx, New York, USA
}

\section{Summary}

Erythrocytes, obtained from a normal adult male and from a patient with Lesch-Nyhan syndrome, were incubated with [8${ }^{14}$ C]adenine and $18-{ }^{14}$ Clhypoxanthine (Table 1). The labeled adenine was utilized to about the same extent for the synthesis of AMP by the normal subject's and the patient's erythrocytes. Deamination of AMP to IMP occurred to about the same extent in both samples. In contrast, hypoxanthine was utilized extensively for INIP synthesis in the normal erythrocyte only. The amount of total label in the IMP was about 100 times that of the Lesch-Nyhan erythrocyte, a consequence of the deficiency of hypoxanthine-guanine phosphoribosyltransferase (HGPRT) activity in the syndrome. No significant labeling of the $\mathrm{AMP}$ occurred. When aliquots of erythrocytes from both sources were incubated with 4-amino-5-imidazolecarboxamide (AICA) and sodium [ ${ }^{14}$ C|formate, extensive labeling of the IMP occurred in normal and in Lesch-Nyhan erythrocytes. The data suggest that AICA serves as a substrate for the adenine phosphoribosyltransferase (APRT) of the Lesch-Nyhan erythrocyte and that the ribotide of AICA, 5'-phosphoribosyl-5-aminoimidazole-4-carboxamide (AICAR), undergoes formylation by labeled $\mathrm{N}^{11}$ formyl tetrahydrofolic acid formed from the reaction of sodium

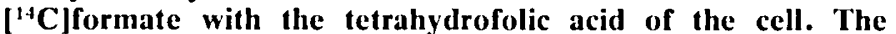
formyl-AICAR undergoes ring closure to IMP by a series of reactions comparable to those described for the normal erythrocyte. When 5-amino-1-ribosyl-4-imidazolecarboxamide (rAICA) and sodium $\left[{ }^{14} \mathrm{C}\right]$ formate were incubated with erythrocyte suspensions, extensive utilization for IMP synthesis was also observed in normal erythrocytes and in erythrocytes from LeschNyhan patients (Table 2). The reaction sequence is somewhat different from that of AICA. rAICA is not a substrate for the purine nucleoside phosphorylase of rabbit or human erythrocytes. The mechanism of $\mathrm{rAICA}$ utilization is visualized as a direct phosphorylation of the ribosyl compound, possibly by the adenosine kinase of the human cell. The ribotide, AICAR, formed by this mechanism, undergoes formylation and ring closure, yielding INIP. The glutamine antagonist, diazooxonorleucine (DON), was added to aliquots of patients' cells incubated with rAICA and sodium $1^{14} \mathrm{C}$ formate. DON is an effective inhibitor of the conversion of IMIP to GMP and its presence in an incubation suspension resulted in a somewhat greater radioactivity of the total cellular IMP.

The extension of the current studies to Lesch-Nyhan cells in culture may serve to assist in the direct evaluation of the regulatory role of IMP in the de novo pathway of purine nucleotide biosynthesis. Because of the substrate requirements of the reactions, the metabolism of AICA and rAICA may also serve to differentiate the roles of purine nucleotides and of phosphoribo- sylpyrophosphate (PRPP) in the pathway regulation. The findings presented also offer a possible therapeutic approach to the early treatment of the disease in the afflicted neonate. The administration of AICA and/or rAICA, possibly supplemented with folic acid and allopurinol, by providing a route to INIP and GMP, with or without concomitant reduction of the elevated PRPP levels, might eliminate, delay, or reduce the development of the symptoms and manifestations of the genetic disease.

\section{Speculation}

The erythrocytes of patients with Lesch-Nyhan syndrome, although lacking the enzymic capacity for conversion of the 6ketopurines, hypoxanthine and guanine, to the corresponding ribonucleotides, can convert AICA and its ribosyl derivative (rAICA) to IMIP by two different reaction mechanisms, thus bypassing the enzymic deficiency. The pathways described should be applicable to studies of regulatory mechanisms in de novo purine nucleotide synthesis and in purine nucleotide interconversions. The imidazole compounds may also have therapeutic application in the early clinical treatment of the syndrome.

The Lesch-Nyhan syndrome is characterized by mental retardation, choreoathetosis, aggressive personality, and a bizarre urge for self-mutilation (8). The X-linked recessive disorder has been associated with the inactivity of a specific enzyme of purine metabolism (23). The deficient enzyme, HGPRT, is primarily responsible in normal cells for reutilization of the 6-ketopurines, hypoxanthine and guanine, which are derived from the breakdown of purine-containing compounds. Since the resultant nucleotides, inosine 5'-phosphate and guanosine 5'-phosphate, serve as allosteric regulators of the initial enzyme of the de novo pathway of purine nucleotide biosynthesis, glutamine phosphoribosylpyrophosphate amidotransferase, the deficiency may account, in part, for the overproduction of purine nucleotides de novo as a characteristic property of the Lesch-Nyhan syndrome (26). The failure to reutilize the purine bases, a consequence of the enzymic deficiency, results in the extensive oxidative metabolism of hypoxanthine and guanine and a pronounced hyperuricemia and hyperuricosuria. It also results in elevated cellular levels of PRPP, the other substrate of the HGPRT reaction (18).

Although there is no significant source of free adenine in humans, the enzyme APRT is present in normal human and Lesch-Nyhan cells and can effect the formation of AMP from administered adenine. The AMP, a regulator of the initial enzyme, could be converted, in part, to IMP and GMP. However, the administration of large amounts of adenine can lead to 
deposition of 2,8-dioxyadenine in the kidneys and a resultant impairment of renal function (20). Nevertheless, in an attempt to enhance the level of an allosteric regulator of glutamine phosphoribosylpyrophosphate amidotransferase, and perhaps to reverse the physical manifestations of the syndrome, several investigators have administered to patients the purine base, adenine, alone or in conjunction with folic acid and allopurinol $(1,20,21)$.

It has been demonstrated in this laboratory that the normal human erythrocyte lacks the capacity for the biosynthesis of the purine nucleotides by the overall de novo pathway. Yet the eell can carry out the final reactions of the pathway (13), utilizing AICA or rAICA as precursors of the nucleotide intermediate, AICAR. Thus, AICAR, formed either by phosphorylation of rAICA or by a phosphoribosyltransferase-catalyzed reaction of AICA and PRPP, can undergo formylation and ring closure to yield IMP in reactions that do not require the intact de novo pathway of purine nucleotide synthesis (Fig. 1).

Since these reactions could serve to bypass the IIGPRT deficiency in the cells of a Lesch-Nyhan patient, this study was undertaken to determine the eapacity of the Lesch-Nyhan erythrocyte to utilize AICA or $\mathrm{AAICA}$ for the formation of INIP in the absence of HGPRT activity.

\section{MATIRIALS ANI) MIETHODS}

Purines, purine nucleotides, and PRPP were purchased from Schwarz/Mann, P-L Biochemicals, Sigma, and Bochringer Mannheim. AICA and rAICA were obtained from Calbiochem. DON was obtained from the Memorial Sloan Kettering Cancer Center, New York. N. Y. Sodium $\left.\right|^{14} C$ ] formate was from Amersham Searle. $\left[8-{ }^{14} \mathrm{C}\right]$ Hypoxanthine and $\left[8-{ }^{11} \mathrm{C}\right]$ adenine were products of Schwarz/Mann.

Venous blood samples, from three patients with Lesch-Nyhan syndrome, were kindly provided by Dr. Joseph Dancis. New York University Medical Center. Patient $J A$ was 12 years of age. Because of the mutilation of his lips and fingers, most of his teeth had been extracted and his elhows were restrained. He had not been receiving allopurinol at the time of the study. Patient $P B$ was a 3-year-old who began to self-mutilate about a year carlier. He had been receiving allopurinol $(100 \mathrm{mg} / \mathrm{day})$. At the time of the study, patient $(W$ was a 10 year old who demonstrated the neurologic manifestations of the syndrome and self-mutilation of lips and fingers. He had been maintained on allopurinol.

Control blood samples were drawn from a normal adult male. All blood samples had been obtained in accord with the Declaration of Helsinki. Whole blood samples, collected in heparin, were centrifuged. Plasma and buffy coat were removed. Washed intact erythrocytes were incubated with appropriate substrates at $37^{\circ}$ for $2 \mathrm{hr}$. At the end of the incubation period, the intact erythrocytes were centrifuged and washed three times with isotonic saline solution. The washed cells were extracted with cold $8 \%$ perchloric acid. The extracts were entrifuged and the supernatant solutions were neutralized with $5 \mathrm{M} \mathrm{K}_{2} \mathrm{CO}_{3}$. After cooling, the potassium perchlorate precipitates were removed by centrifugation. Carrier IMPP (0.12 $\mu \mathrm{mol}$ in $20 \mu \mathrm{l}$ solution) and aliquots of each extract $(60 \mu \mathrm{l})$ were applied in $5 \mu$ alicuots, to squares $\left(2.25 \mathrm{~cm}^{2}\right)$ of diethylaminocthyl (DEAE)-cellulose patper (Whatman DE81). The DEAE-paper binds nucleotides, facilitating the removal of free purines, nucleosides, and excess sodium ["1 C]formate.

Each dried square was washed using the method of Silvers et al. (24), and then sewn on to a polyethyleneimine (PEI)-cellulose thin layer chromatographic plate (Brinkmann Instruments, Inc.). Chromatography was carried out in $50 \mathrm{ml} 0.5 \mathrm{M} \mathrm{LiCl}_{2}$ and $50 \mathrm{ml} 2 \mathrm{M}$ formic acid.

Compounds on the dried plate were identified and eluted from the scraped cellulose powder with $0.1 \mathrm{~N} \mathrm{HCl}$. The identities were verified spectrophotometrically. Aliguots $(0.1 \mathrm{ml})$ of the cluates were assayed for radioactivity in a Packard scintillation counter. Data are expressed as calculated total radioactivity of the nucleoticle per ml erythrocyte.

\section{RESULTS}

Erythrocytes, obtained from a normal adult male and those obtained from a patient with Lesch-Nyhan syndrome were incubated with $\left[8-{ }^{14} \mathrm{C}\right] \mathrm{adenine}$ and $[8-1+\mathrm{C}]$ hypoxanthine (Table 1). The labeled ade nine was utilized to about the same extent for the synthesis of AMP by the normal and the patient's erythrocytes. Deamination of ANP to INIP oceurred to about the same extent in both samples. The formation of $A M P$ is in accord with the known presence of an active APRT in normal and Lesch-Nyban erythrocytes. In contrast, hypoxanthine was utilized extensively for IMP synthesis in the normal erythrocyte only. The amount of total label in the IMP was about 100 times that of the LeschNyhan erythrocyte, a consequence of the deficiency of HGPRT activity in the symdrome. No significant labeling of the ANP occurred.

When aliquots of erythrocytes from both sources were incubated with t-amino-5-imidazolecarboxamide and sodium ${ }^{14} \mathrm{C} \mid \mathrm{f}$ ormate, extensive labeling of the INIP occurred in normal and in Lesch-Nyhan erythrocytes. The data suggest that AICA serves as a substrate for the APRT of the Lesch-Nyhan erythrocyte and that the ribotide of $A I C A(A I C A R)$ undergoes formylation by labeled $N^{11}$-formyl tetrahydrofolic acid formed from the reaction of sodium [ "C] formate with the tetrahydrofolic acid of the cell. The formyl-AICAR undergoes ring closure to IMP by a series of reactions comparable to those described for the normal erythrocyte.

The absence of appreciable label in the AMP obtained from the hypoxanthine and AICA experiments suggests that the Lesch-Nyhan erythrocyte and the normal human erythrocyte resemble each other in their inability to convert IMP to AMP (13), a result of the absence of adenylsuccinate synthetase activity (10)

The finding that AICA and sodium [ ${ }^{14} \mathrm{C}$ formate were utilized for IMP synthesis in the Lesch-Nyhan erythrocyte was extended to the erythrocytes of two additional patients (Table 2). Although the extent of labeling of the IMIP was somewhat variable, considerable labeling did occur, in contrast to the erythrocyte samples incubated with sodium [1"C]formate alone. This reflected the inability of mature human erythrocytes to synthesize purine nucleotides by the de novo pathway. The small amounts

Table 1. Utilization of adenine, hypoxanthine, and 4-amino-5-imidazolecarboxamide (AICA) for synthesis of purine nucleotides by mature erythrocytes from paticnt with Lesch-Nyhan syndrome and normal adult male'

\begin{tabular}{|c|c|c|c|c|c|c|}
\hline \multirow[b]{3}{*}{ Subject } & \multicolumn{6}{|c|}{ Total cpm/ml cells } \\
\hline & \multicolumn{2}{|c|}{$\left[8-{ }^{14} \mathrm{C}\right]$ Adenine $5.0 \mu \mathrm{Ci}, 1.6 \mu \mathrm{mol}$} & \multicolumn{2}{|c|}{$\begin{array}{c}{\left[8-{ }^{14} \mathrm{C}\right] \text { Hypoxanthine } 5.0 \mu \mathrm{Ci}, 1.6} \\
\mu \mathrm{mol}\end{array}$} & \multicolumn{2}{|c|}{$\begin{array}{c}\text { Sodium }\left[\mathrm{C}^{14}\right] \text { formate } 5.0 \mu \mathrm{Ci}, 3.1 \\
\mu \mathrm{mol} ; \mathrm{AICA}, 1.5 \mu \mathrm{mol}\end{array}$} \\
\hline & AMP isolated & IMP isolated & AMP isolated & IMP isolated & ANP isolated & IMP isolated \\
\hline Normal $(B L)$ & 7,040 & 2,750 & 417 & 268,900 & 541 & 43,440 \\
\hline Paticnt $(J A)$ & 7,290 & 2,300 & 42 & 2,960 & 791 & $45,(120$ \\
\hline
\end{tabular}

${ }^{1}$ Aliquots of washed crythrocytes $(0.85 \mathrm{ml})$ were incubated for $2 \mathrm{hr}$ at $37^{\circ}$ in equal volumes of isotonic sodium phosphate buffer (pH 7.4 ) containing glucose $(15 \mu \mathrm{mol})$. Carrier IMP was added. Nucleo.ides were isolated, purified, and assayed for radioactivity. 
Table 2. Utilization of 4-amino-5-imidazolecarboxamide' (AICA) and its ribosyl derivative ( $\mathrm{A} A \mathrm{ICA}$ ) by mature erythrocytes from patients with Lesch-Nyhan syndrome and normal adult male'

Total cpm/ml cells

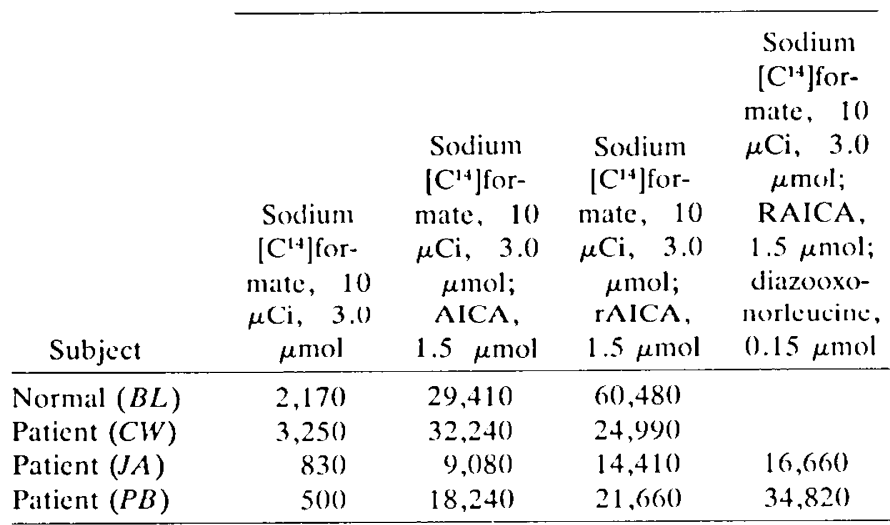

1 Alicuots of washed erythrocytes $(1.0 \mathrm{ml})$ were incubated for $2 \mathrm{hr}$ at $37^{\circ}$ in equal volumes of isotonic sodium phosphate huffer ( $\mathrm{pH} 7.4$ ) containing glucose $(15 \mu \mathrm{mol})$. Carrier IMP was added. Total IMP was isolated, purified, and assayed for radioactivity.

of label in the latter IMP fractions are based on values only slightly above background radioactivity and could be attributed to the small number of reticulocytes in the preparation.

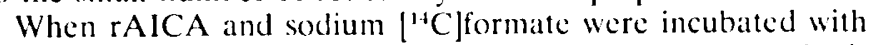
erythrocyte suspensions, extensive utilization for IMP synthesis was also observed in normal erythrocytes and in erythrocytes from Lesch-Nyhan patients. The reaction sequence is somewhat different from that of AICA. rAICA is not a substrate for the purine nucleoside phosphorylase of rabbit or human erythrocytes $(9,11)$. The mechanism of rAICA utilization is visualized as a direct phosphorylation of the ribosyl compound, possible by the adenosine kinase of the human cell (12). The ribotide AICAR, formed by this mechanism, undergoes formylation and ring closure, yiclding IMP.

The glutamine antagonist, DON was added to aliquots of patients' cells incubated with rAICA and sodium $\left[{ }^{14} \mathrm{C}\right]$ formate. DON is an effective inhibitor of the conversion of IMP to GMP (11) and its presence in an incubation suspension resulted in a somewhat greater radioactivity of the total cellular IMP. This effect was also observed when erythrocytes from a normal adult male were incubated with AICA and sodium $\left[{ }^{14} \mathrm{C}\right]$ formate in the presence and absence of DON.

The purine nucleotides of the perchloric acid extract from patient $J A$ were hydrolyzed and the radioactivity of the purines was determined. No significant labeling of the isolated adenine was detected, even in the presence of sufficient DON to inhibit the conversion of IMP to GMP. Hypoxanthine, obtained from the labeled IMP, was appreciably labeled in accord with the data of Table 1 .

\section{DISCUSSION}

Despite the numerous studies that have been concerned with the biochemistry of the Lesch-Nyhan syndrome, the causal relationship between the enzymic deficiency and the behavioral and physical manifestations of the disease remains intriguing and elusive. A number of interesting observations may be pertinent to its etiology. It has been found that, in the normal human, the major site of HGPRT activity is in the brain (19). The enzymic deficiency in the brain of the Lesch-Nyhan individual and a resultant increased activity of the de novo pathway could lead to a diminution of brain glutamine or a related metabolite (4), since 2 molecules of the amino acid are required for each molecule of IMP formed, and a third molecule is required for the conversion of IMP to GMP. Furthermore, it has been reported that the activity of IMP dehydrogenase, an enzyme on the pathway to
GMP, is increased in Lesch-Nyhan patients (17). The stimulation of de novo synthesis, which could be a consequence of clevated PRPP levels (18), may also affect the cellular folates of all tissues $(6,15)$. The low level of glutanine phosphoribosylpyrophosphate amidotransferase in mammalian brain suggests a dependence upon HGPRT for purine nucleotide formation(5).

The present study provides experimental evidence for enzymic activities within the Lesch-Nyhan erythrocyte that can lead to a metabolic bypass of the HGPRT deficiency in the genetic disease. The presence of APRT and of a kinase that can phosphorylate rAICA serves to accomplish the synthesis of IMP from endogenously supplied precursors. The reactions have been summarized in Figure 1.

The first reaction sequence occurs because AICA can serve as a substrate for the APRT of normal and Lesch-Nyhan erythrocytes (25). Since the compound is an inhibitor of HGPRT (7), a more effective utilization would not be expected in the normal erythrocyte which contains both HGPRT and APR'T activities.

The second reaction sequence is dependent upon the presence of a kinase, possibly adenosine kinase (12), that can phosphorylate rAICA. The kinase activity has previously been found in yeast, pigeon liver, and normal human and rabbit erythrocytes $(2,11,13)$.

Since GMP is readily formed in the Lesch-Nyhan cell from IMP, the demonstration of a bypass mechanism for the formation of IMP in the erythrocyte of the patient also implies a pathway to GMP, thereby overcoming the total qualitative defect of the disease, without the involvement of the overall de novo pathway and the problem of an overactive series of reactions.

An enhancement of IMP and GMP levels in the Lesch-Nyhan cell and a direct study of their regulatory roles in vitro have not been undertaken to date, for a number of reasons. Exogenous nucleotides, per se, cannot permeate the cell membrane. Although purine bases and their nucleosides enter the cell, conversion of the 6-ketopurines to the nucleotides requires HGPRT. Furthermore, in the absence of an appreciable ability of mammalian cells to phosphorylate inosine and guanosine, the 6ketopurine nucleosides are not converted to the nucleotides (3, 16).

The mammalian erythrocyte is unable to synthesize the purine nucleotides by the de novo biosynthetic pathway and hence the bypass mechanism demonstrated to exist within that cell can not be applied to a study of the regulation of the de novo pathway in either the normal or Lesch-Nyhan erythrocyte. However, the mechanism is applicable to studies in other Lesch-Nyhan cells and tissues in which de novo purine nucleotide synthesis may be

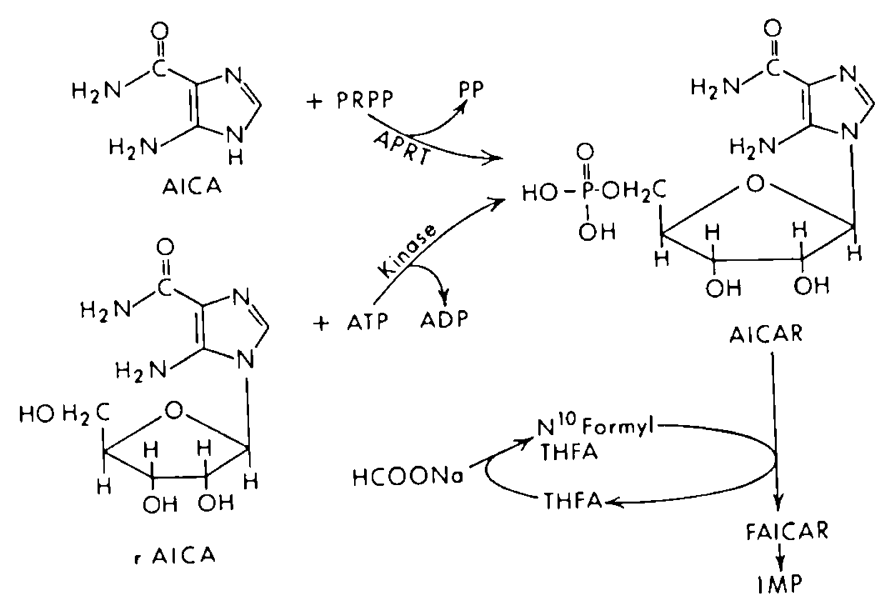

Fig. 1. Enzymic capacity of the human erythrocyte for IMP synthesis from 4-anino-5-imidazolecarboxamide $(A I C A)$ and its ribosyl derivative $(r A I C A)$. PRPP: Phosphoribosylpyrophosphate; PP: pyrophosphate; AICAR: 5'-phosphoribosyl-5-aminoimidazole-4-carboxamide; FAICAR: formyl-AICAR; THFA: tetrahydrofolate. 
poorly regulated. The extension of the current studies to LeschNyhan cells in culture may serve to assist in the direct evaluation of the regulatory role of $1 \mathrm{MP}$ in the de novo pathway of purine nucleotide biosynthesis. Because of the substrate requirements of the reactions, the metabolism of AICA and $\mathrm{rAICA}$ may also serve to differentiate the roles of purine nucleotides and of PRPP in the pathway regulation. Although the clinical problems associated with the oxidation of endogenous hypoxanthine and guanine to uric acid remain, the ramifications of poorly regulated de nowo synthesis and possible substrate and cofactor depletion may be amenable to further evaluation.

The findings presented also offer a possible therapeutic approach to the carly treatment of the discase in the afflicted neonate. The administration of AICA and/or rAICA, possibly supplemented with folic acid and allopurinol, by providing a route to IMP and GMP, with or without concomitant reduction of the elevated PRPP levels, might eliminate, delay, or reduce the development of the symptoms and manifestations of the genetic disease. Several investigators have studied the metabolism and excretion of the imidazole compounds in the normal human. The studies suggest that oral administration of the compounds is not accompanied by toxic effects at the levels utilized in those studies $(14,22,27)$.

\section{RHFEREN(ISS ANI) NOTES}

1. Benke, P. J., Herrick, N., Smitten, L., Aradine, C., Lacssig, R., and Wolcott, G. J.: Adenine and folic acid in the Lesch-Nyhan syndrome. Pediat. Res., 7: 729 (1973).

2. Flaks, J. G., Erwin, M. J., and Buchanan, J. M.: Biosynthesis of the purines. XVI. The synthesis of adenosine 5 -phosphate and 5 -amino-4-imidazolecarboxamide ribotide by a nucleotide pyrophosphorylase. J. Biol. Chem., 228: 201 (1957).

3. Friedmann, T., Seegmiller, J. E., and Subak-Sharpe, J. H.: Evidence against the existence of guanosine and inosine kinases in human fibroblasts in tissue culture. Exp. Cell Res. 56: 425 (1969).

4. Ghadimi, H., Bhalla, C. K., and Kirchenbaum, D. M.: The significance of the deficiency state in Lesch-Nyhan disease. Acta Pacdiat. Scand., 59: 233 (1970).

5. Howard, W. J., Kerson, L. A., and Appel, S. H.: Synthesis de novo of purines in slices of rat brain and liver. J. Neurochem., 17: 121 (1970).

6. Kelley, W. N., Greene, M. L., Rosenbleom, F. M., Henderson, J. F., and Secgmiller, J. E.: Hypoxanthine-guanine phosphoribosyltransferase in gout. Ann. Intern. Med., 70: 155 (1969).

7. Krenitsky, T. A., Papaioannou, R., and Elion, G. B.: Human hypoxanthine phosphoribosyltransferase. I. Purification, properties and specificity. J. Biol. Chem., 244: 1263 (1969).

8. Lesch, M., and Nyhan, W. L.: A familial disorder of uric acid metabolism and central nervous system function. Amer. J. Med., 36: 561 (1964).

9. Lewis, A. S., and Lowy, B. A.: Unpublished observation.

10. Lowy, B. A., and Dorfman, B.: Adenylosuccinase activity in human and rabbit erythrocyte lysates. J. Biol. Chem., 245: 30.43 (1970).

11. Lowy, B. A., and Williams, M. K.: The presence of a limited portion of the

Copyright (1) 1977 International Pediatric Rescarch Foundation, Inc. pathway de now of purine nucleotick biosynthesis in the rabbit erythrocyle in vitro. J. Biol. (hem. 235: 2924 (1960).

12. Lowy, B. A., and Williams, $\mathbb{M}$. K.: Studies on the metabolism of atenosine and adenine in stored and fresh human erythrocytes. Blood, 27: 623 $(1966)$.

13. Lowy, B. A., Williams, M. K., and London, I. M1.: Inzymatic deficiencies of purine nucleotide synthesis in the human erythrocyte. J. Biol. Chem., 237: $1622(1962)$

14. McGeer, P. L., McGere, E. G., and Griffin, M. C.: Excretion of 4-imino-5imidazole-carboxamide in human urine. (antad. J. Biochem., 39: 591 (1961).

15. Newcombe, D. S.: The urinary excretion of aminomidatelecarboxamide in the Lesch-Nyhan syndrome. Pediatries, 46: 508 (1970).

66. Payne, M. R., Dancis, J., Berman, P. H., and Batis, M. E.: Inosine hinase in leucocytes of Lesch-Nyhan pationts. Exp. (ell Res., 59: 489 (1970).

17. Peblke, D. M. McDonald, J. A. Holmes, E. W.. and Kelley, W. N.: Inosinic acid dehydrogenase activity in the Lesch-Nyhan syndrome. J. Clin Invest. 51: 1398 (1972).

18. Rosenbloom, F. M., Henderson, J. F., Caldwell, I. C., Kelley, W. N., and Seegmiller, J. E.: Biochemical bases of accelerated purine biosynthesis de nov'o in human fibroblasts lacking hypoxanthine-guanine phosphoribosyltransferase. J. Biol. Chem.. 243: $1166(1968)$.

19. Rosenbloom, F. M., Kelley, W. N., Mtiller, J., Henderson, J. F., and Seegmiller, J. E: : Inherited disorder of purine metabolism: Correlation between central nervous system dysfunction and biochemical defects. J. Amer. Med. Ass., 202: 175 (1967).

20. Schulman, J. D., Greene, M. L., Fujimoto, W. Y., and Secgmiller, J. E.. Adenine therapy for Lesch-Nyhan syndrome. Pediat. Res., 5: 77 (1971).

21. Secgmiller, J. E.: Lesch-Nyhan syndrome management and treatment: Seminars on Lesch-Nyhan ssndrome. Fed. Proc., 27: 1097 (1968).

22. Secemiller, J. E., Laster, L., and Stetten, D., Jr.: Incorporation of 4-amino-5imidazolecarboxamide-4-( ${ }^{13}$ into uric acid in the normal human. J. Biol. Chem., 216: 6.53 (1955).

23. Seegmiller, J. E., Rosenbloom, F. M., and Kelly, W. N.: Enzyme defect associated with a sex-linked human neurological disorder and excessive purine synthesis. Science, 155: $1682(1967)$.

24. Silvers, D. N., Cox, R. P., Balis, M. E., and Dancis, J.: Detection of the heterogygote in lesch-Nyhan discase by hatr-root analysis. New Ingl. J. Med., 286: 390 (1972)

25. Thomas, C. B: Arnold, W. J., and Kelley, W. N.: Human adenine phosphoribosyltransferase. Purification, subunit structure, and substrate specificity. J. Biol. Chem., 248: 2529 (1973).

26. Wyngaarden, J. B., and Ashton, D. M.: The regulation of activity of phosphoribesylpyrophosphate amidotransferase by purine ribonucleotides: $A$ potential feedback control of purine biosynthesis. J. Biol. Chem., 234: 1492 (1959).

27. Wyngaarden, J. B., Seegmiller, J. E., Laster, L., and Blair, A. E.: Utilization of hypoxanthine, adenine and 4-amino-5-imidazolecarboxamide for uric acid synthesis in man. Metab. Clin. Lxp., 8: 455 (1959).

28. This work was presented in part at the Second International Symposium on Purine Metabolism in Man, Baden/Vienna, Austria, Junc 1976.

29. This work was supported by Grant AM 17622 from the National Institute of Arthritis, Metabolism and Digestive Diseases of the National Institutes of Health.

30. Requests for reprints should be addressed to: B. A. Lowy, Ph.D., Department of Biochemistry, Albert Einstein College of Medicine, Bronx, N. Y. 10.461 (USA).

31. Received for publication June 24, 1976.

32. Accepted for publication October 20,1976 